Triona McNamara, BDS, FDS, DOrth, Carroll-Ann Trotman, BDS, MA, Antonia Hewson, BDS, Matthew Walshe, BDS

\title{
Facial developmental vascular anomalies
}

\author{
Developmental vascular anom \\ alles of the head and neck may give \\ rise to profound esthetic problems \\ that are a challenge to treat. These \\ vascular anomalies may also be of \\ significance in dentistry, particularly \\ where extractions have to be carried \\ out. This brief report describes a \\ number of children who presented \\ with different variations of these \\ anomalles.
}

$\mathbf{T}$ here are two major categories of developmental vascular anomalies, hemangiomas and vascular malformations. Hemangiomas are frequently referred to in textbooks and articles as benign tumors and are recorded as "the most common tumors of infancy." ${ }^{1}$ Pathologists, however, do not consider hemangiomas true benign tumors and define them as vascular hamartomas. Unlike a true benign tumor, a hamartoma is a pathological entity in which tissues of a particular part of the body are arranged haphazardly with an excess of one or more of its components. A hamartoma also has no capsule, and ceases to enlarge with cessation of growth of the patient.

Differentiating a hemangioma from a developmental vascular malformation is important. Clinically, the natural history of the vascular anomaly may be the determining factor. A hemangioma is usually not present at birth. It characteristically proliferates very rapidly, at a much greater rate than the infant's growth, during the first few weeks of life, and then spontaneously involutes. A developmental vascular malformation, however, is present at birth. It tends to have a persistent colored hue, depending on its arterial, venous, capillary, or lymphatic components, grows with the birth of the child, and does not regress significantly.

The incidence of hemangiomas is reported to be $1 \%$ to $2.6 \%$, and the male-to-female ratio is $1: 3$. The most common site is the head and neck region $(60 \%)$, followed by the trunk $(25 \%)$, and then the limbs ( $15 \%)$. The greater percentage distribution of these lesions in the face may be influenced by the higher reporting in those instances where facial lesions are found. Cutaneous hemangiomas are superficial (capillary) in approximately $60 \%$ of cases and deep (cavernous) in $15 \%$ of cases.

Developmental vascular anomalies usually occur sporadically and without any genetic basis; however, they may be an important component of diseases with a strong genetic predisposition. For example, hereditary hemorrhagic telangiectasia arises due to arteriolar-venular anastomoses. It is inherited as an autosomal dominant trait and generally becomes apparent at puberty. Sturge-Weber syndrome is characterized by aberrant vasculature of the face, skin, eyes, and meninges. The etiology is unknown and the incidence sporadic. The purplish-red nonelevated naevus flammeus characteristic of this syndrome generally follows the distribution of the trigeminal nerve, but in some instances the lesion. may extend onto the neck, chest, and back. Involvement of the arachnoid and pia mater occurs in this syndrome, which may lead to the development of epilepsy, hemipareses, or mental retardation. Intracranial calcification is visible radiographically in approximately $75 \%$ of cases, and seizures can be observed in $90 \%$ of cases. Facial hemangiomas are also associated with syndromes such as Kasabach-Merritt syndrome, Maffucci's syndrome, Bean's syndrome, and Beckwith's syndrome. ${ }^{1-5}$

\section{Case reports}

This is a description of five children who presented for orthodontic 

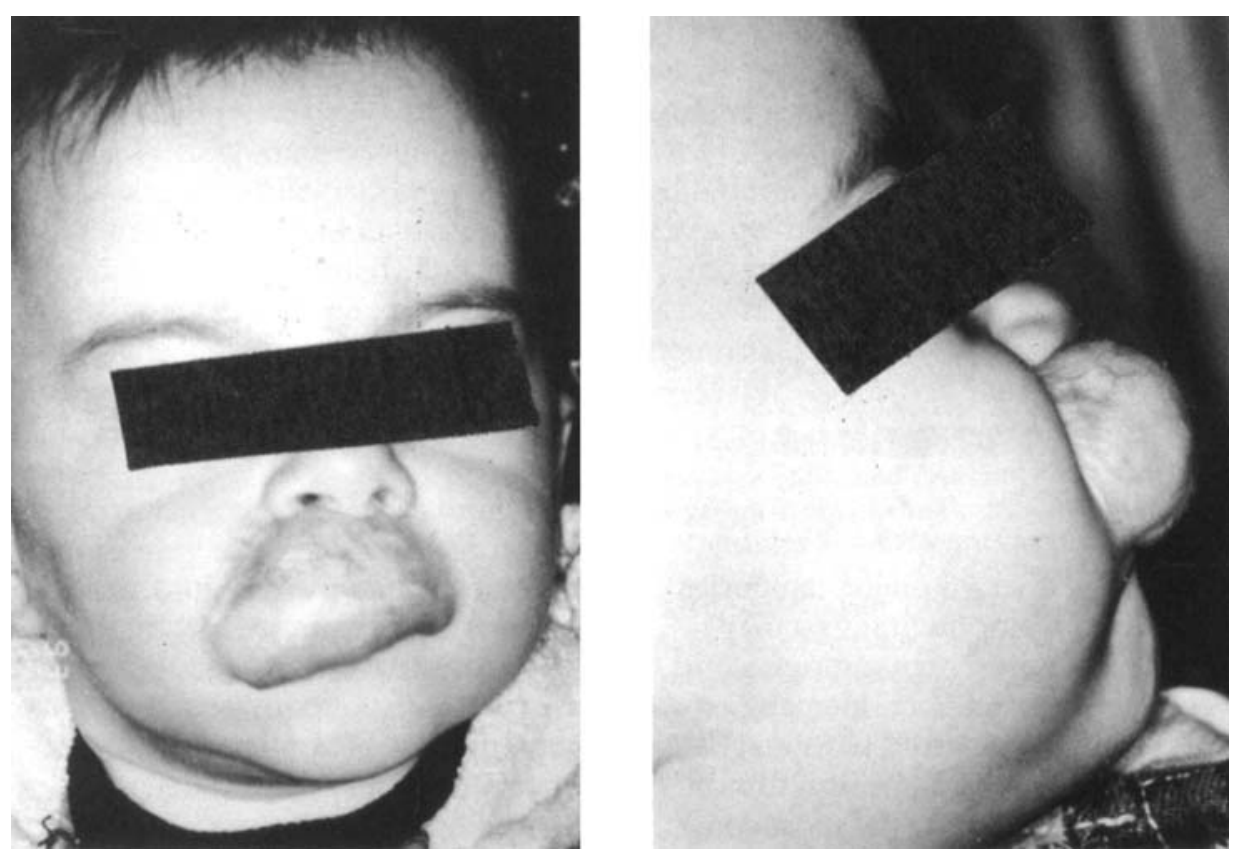

Figures 1 \& 2. Case 1: Frontal and lateral views of the enlarged cavernous hemangioma of the upper lip.

assessment. Cases 1, 2, and 3 have findings that would classify them as facial hemangiomas. Cases 4 and 5 are consistent with facial vascular malformations. Case 5 also has the distribution seen in Sturge-Weber syndrome, but without confirmation

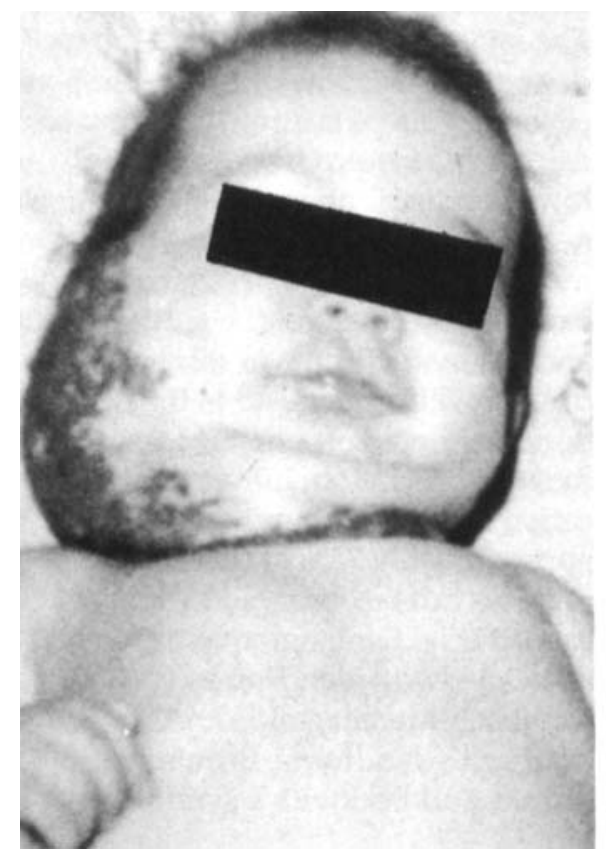

Figure 3. Case 2: Infant, age 3 years, with the cavernous hemangioma at maximum size. of cranial involvement by the medical team. None of the patients had a family history of any developmental vashad an anencephalic sibling brother who died at birth. Excluding Case 3, no life-threatening consequences

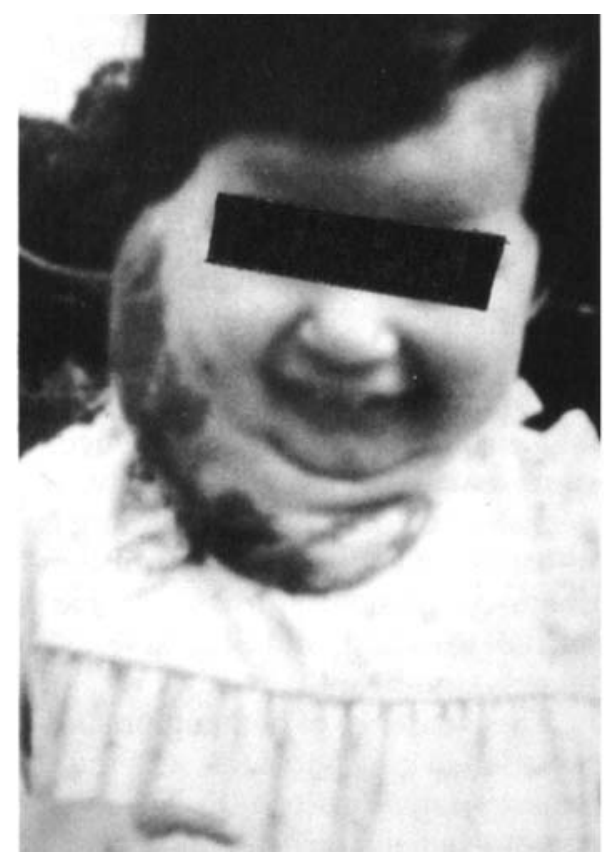

Figure 4. Case 2: Same child, now 5 years, showing the beginnings of the involution phase of the hemangioma. cular abnormalities, although Case 5 have arisen due to the presence of these vascular lesions. Three of the children had lesions involving soft tissues only, and in the cases with bony involvement, the lesions were confined unilaterally to the maxilla.

\section{Case 1}

The first case report concerns a boy who presented for examination at two years of age. The cavernous hemangioma of the upper lip was not present at birth, but enlarged rapidly within the first few months of life (Figs 1 and 2). Rapid growth of this lesion has now ceased; however, due to the gross esthetic disfigurement and distortion of his nose, he has undergone early surgery. Large mattress-type sutures were inserted into the nasolabial fold region in order to contain the lesion during its proliferative phase, and he is currently undergoing phased laser treatment.

Dentally, there were no initial complications. The deciduous dentition erupted normally. Once the labial hemangioma became evident, however, there was distortion and molding of the premaxillary area, with a resultant Class III incisor relationship. No treatment is indicated at

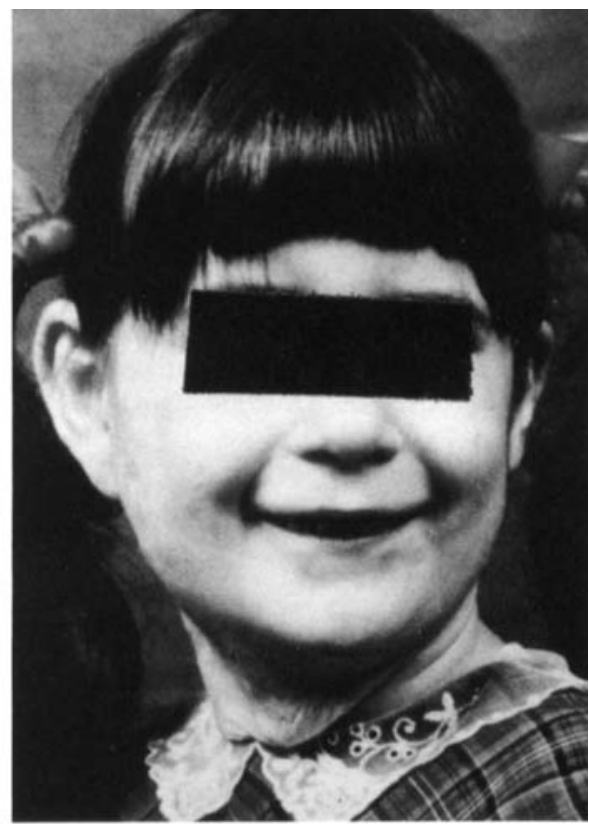

Figure 5. Case 2: Same child, now 7 years, showing almost total involution of the hemangioma and excess tissue in the neck region. 
this time. Preventive dental advice has been given, and the patient will be monitored on an on-going basis.

\section{Case 2}

The second case concerns an 11year-old girl who presented for orthodontic assessment. Her facial lesion was an extensive cavernoustype hemangioma that extended from her right cheek to the right submandibular region. It was not present at birth. The first evidence of the lesion, a thin persistent red line on her left cheek, was noted by the patient's mother at two weeks of age. This 'red line' enlarged rapidly to an enormous size and to such an extent that it led to gross facial disfigurement and interfered with the child's head posture for some time (Figs 3, 4, and 5).

At three years of age, the cavernous hemangioma had reached its maximum size. At five years, the involution phase began, and by the age of seven years, there was practically total involution of the hemangioma. This involution resulted in a dramatic improvement in the patient's appearance, but the redundant skin overlying the lesion required attention (Fig 5). Surgery was carried out to eliminate this excess tissue. The patient's current facial appearance may be seen in Figs 6 and 7, showing the dramatic esthetic improvement. Further limited laser surgery is planned but only to reduce persistent red hemorrhagic patches.

The dental condition was not related to the facial hemangioma. The central maxillary incisors were avulsed (Fig 8) following trauma. The lateral incisors had drifted mesially and had a poor prognosis because of severe root resorption. Since the hemangioma did not involve the maxilla, routine dental treatment could be carried out. The lateral incisors were extracted and a temporary prosthesis fitted. Long-term dental treatment will be carried out when dental development is completed.

\section{Case 3}

The third case concerns a 16-year-old girl who presented for orthodontic
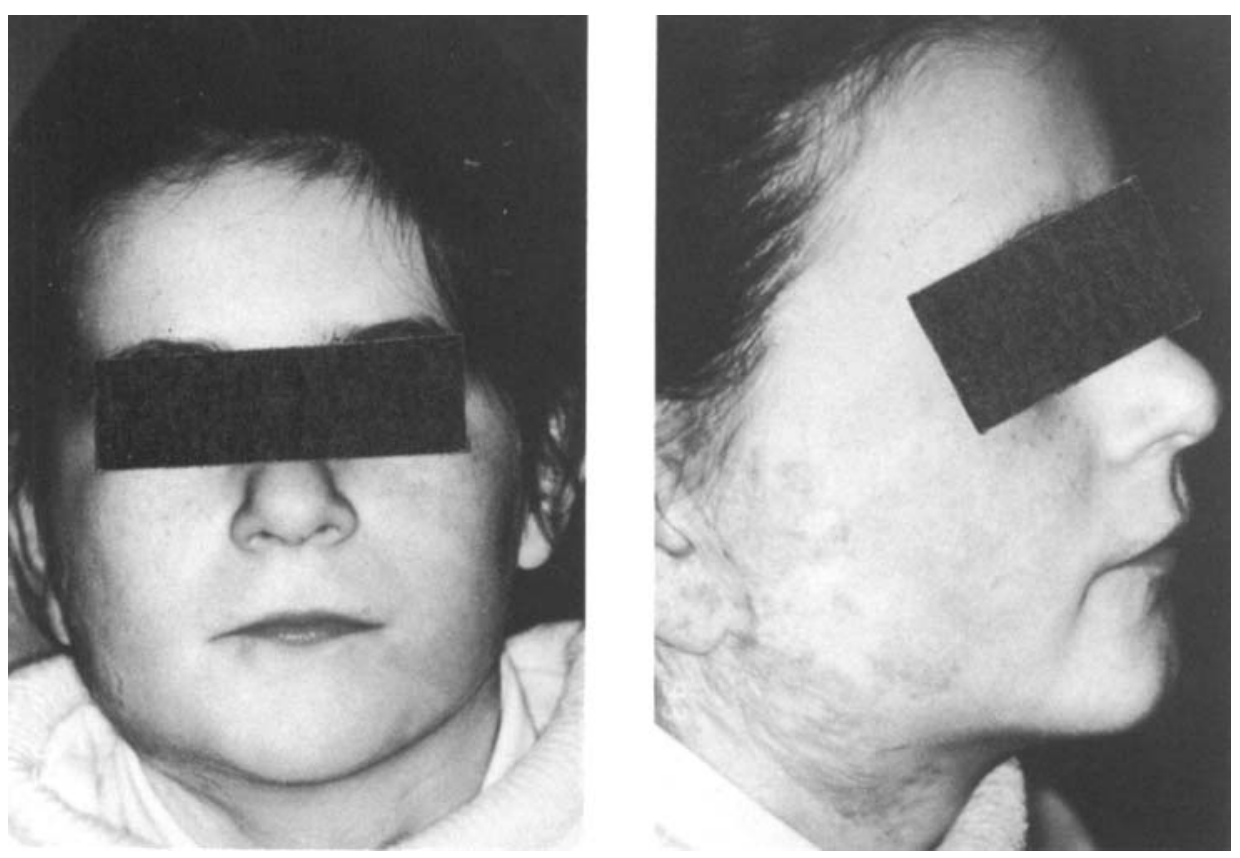

Figures 6 \& 7. Case 2: Frontal and lateral views of the patient after the removal of excess tissue in the neck region.

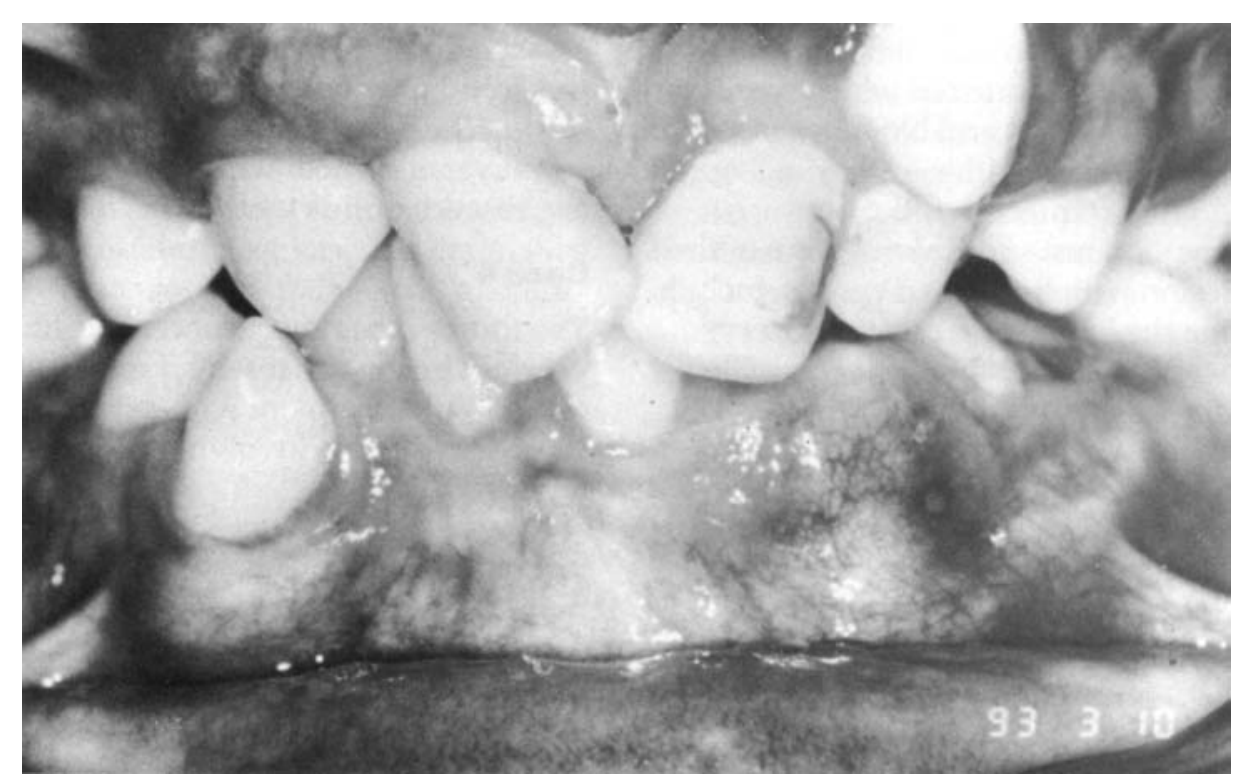

Figure 8. Case 2: Intra-oral view showing avulsed maxillary central incisors with maxillary lateral incisors in the central incisor position.

treatment. She had a cavernous hemangioma involving the left maxillary region, but the lesion was barely visible at the time she presented for orthodontic treatment (Fig 9).

Intraorally, there was no evidence of the lesion (Fig 10). Clinically, the bony form of the maxilla was normal, and the mucosa was not involved.
The cavernous hemangioma of the left maxilla was diagnosed at four years of age, following a dental procedure that led to a major bleed.

There had been a childhood history of recurrent spontaneous bleeds from approximately 18 months of age which were managed by local measures. Following a dental visit, how- 

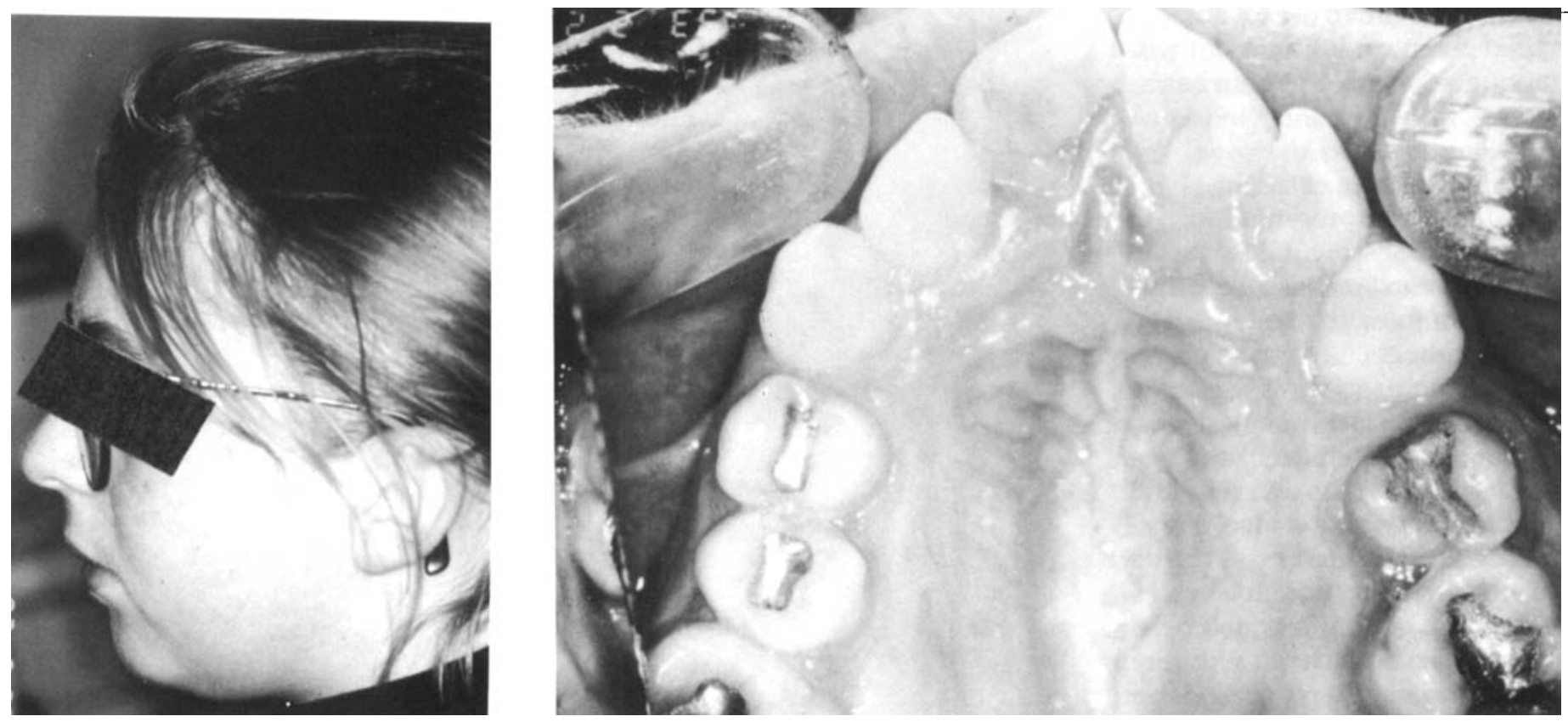

Figures 9 \& 10. Case 3: Right lateral facial and maxillary palatal views showing no evidence of any lesions.

ever, the lesion was 'lanced,' and a major bleed occurred which required hospitalization and blood transfusions.

At seven years, following angiograms, surgical resection of the lesion with ligation of vessels, including the left greater palatine artery, was carried out. No further problems arose again until the patient was 12 years, when a spontaneous bleed occurred which was controlled by cryosurgery. There have been no subsequent incidents of hemorrhage, but due to the long history of bleeds, the patient continues to be monitored by her plastic surgeon on an annual basis.

Because of the maxillary cavernous hemangioma, dental procedures are minimized to ensure that the patient is not put at risk of further hemorrhage. Restorations of teeth in the affected dental quadrant were carried out under local anesthesia. Limited orthodontic treatment is under way at this time, and to date there have been no complications. Minimal forces have been applied, and fortunately, because a premolar was absent in the affected quadrant, orthodontic extractions were not nec- essary. From the medical history, a tooth bud may have been removed during the patient's first operation (at seven years), which would explain the absence of this tooth.

\section{Case 4}

The fourth case report concerns a 12year-old girl who presented for orthodontic treatment with extensive 'port-wine' stains involving the posterior cranium, posterior auricular region, neck, and extending onto the face (Figs 11, 12, and 13). It was present from birth and did not regress.

There were no dental complications. The lesion was confined to skin. Routine orthodontic extractions had already been carried out, and completion of treatment with fixed appliance therapy has begun. The patient has opted to avoid any treatment of the vascular malformation, since the major part of the lesion can be camouflaged by her hair.

\section{Case 5}

The fifth case report concerns a 14year-old girl who presented for orthodontic treatment with a facial hemangioma whose distribution is similar to that seen in Sturge-Weber syndrome. The ophthalmic and maxillary divisions of the trigeminal nerve were involved. The vascular lesion was present from birth, and extended unilaterally from the upper lip on the left side of the face to the top of the cranium, with ocular involvement on that side (Figs 14 and 15). Bony and mucosal involvement of the left quadrant of the maxilla was noted. The patient had normal intelligence. There was no history of epilepsy, and no calcified deposits were noted on routine cephalometric radiographs.

There was a marked Class II division I malocclusion with extensive crowding in both arches. Orthodontic extractions proved unavoidable. Due to the risk of hemorrhage in the affected maxillary quadrant, extractions of the four first premolars was carried out in a hospital environment under local anesthesia. Fixed appliances were utilized to treat the malocclusion, and to date there have been no serious complications.

Regarding the management of the facial anomaly, this patient is currently attending a plastic surgeon on a regular basis, and is undergoing phased laser treatment. Adding to 

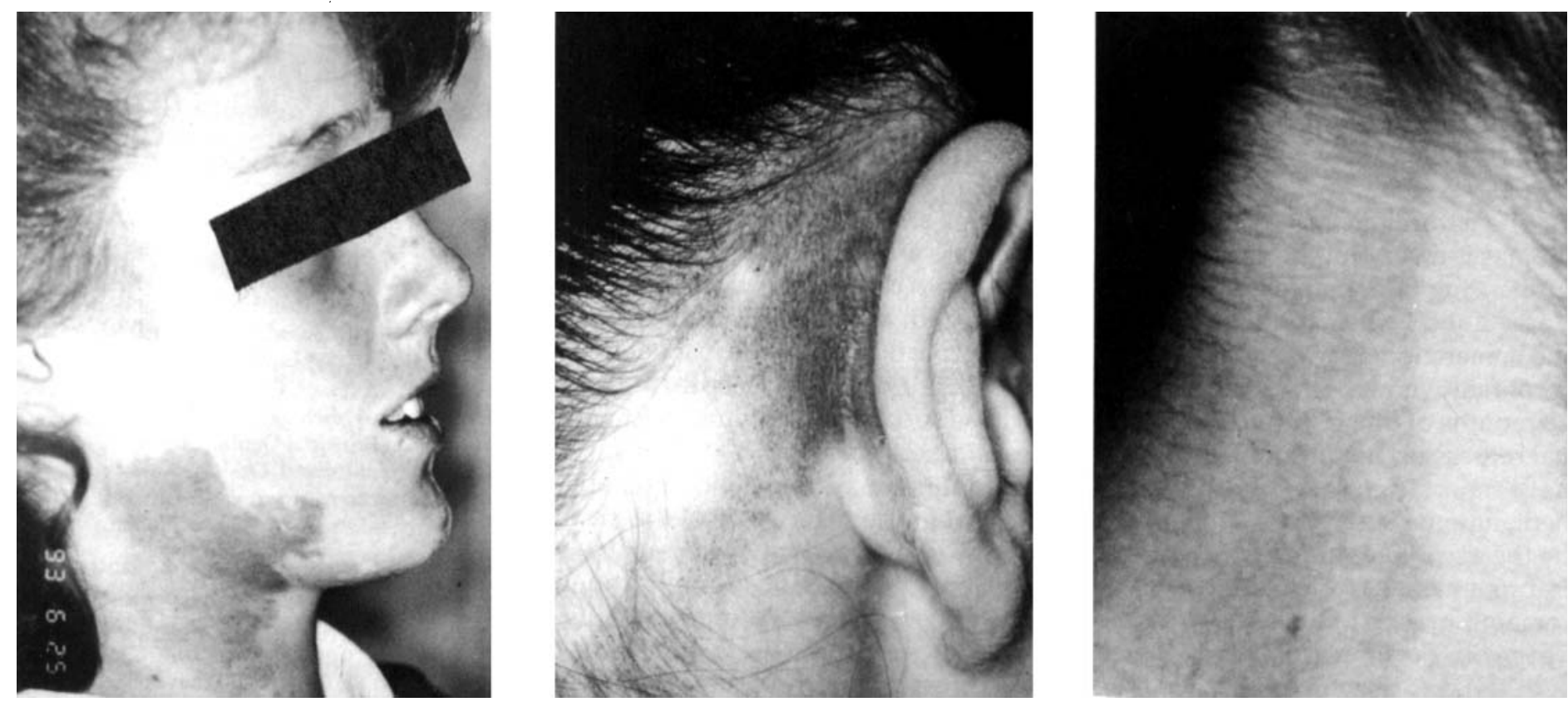

Figures 11, 12 \& 13. Case 4: Facial views showing "port-wine" stain extending onto the neck, face, and the posterior cranial and auricular regions.

the difficulties in treating this case, the patient sustained a bicycle accident at age seven that led to bleeding into the left side of her upper lip, resulting in its current enlarged size.

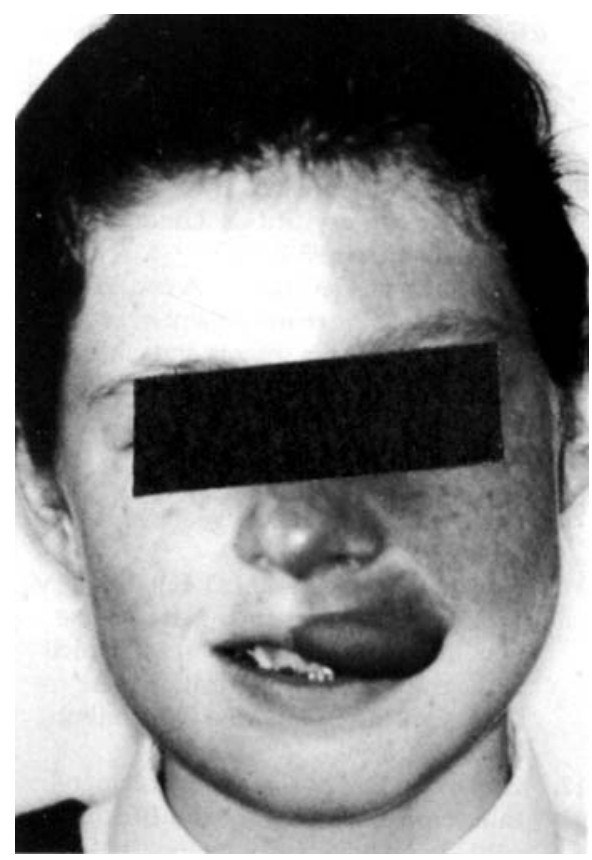

Figure 14. Case 5: Frontal facial view showing hemangioma extending unilaterally from the upper lip on the left side of the face to the top of the cranium.

\section{Discussion}

Management of developmental vascular anomalies is very varied, and whenever possible, minimal treatment is advisable. Most vascular anomalies are cutaneous, remain small, and are harmless; however, a few may grow to alarming size, and depending on their location may give rise to lifeendangering complications such as deformation or obstruction of vital organs, serious bleeding, or sepsis.

A variety of therapeutic modalities exists, ranging from steroid therapy, sclerosing agents, cryosurgery, and surgical excision with or without ligation of vessels, to embolization

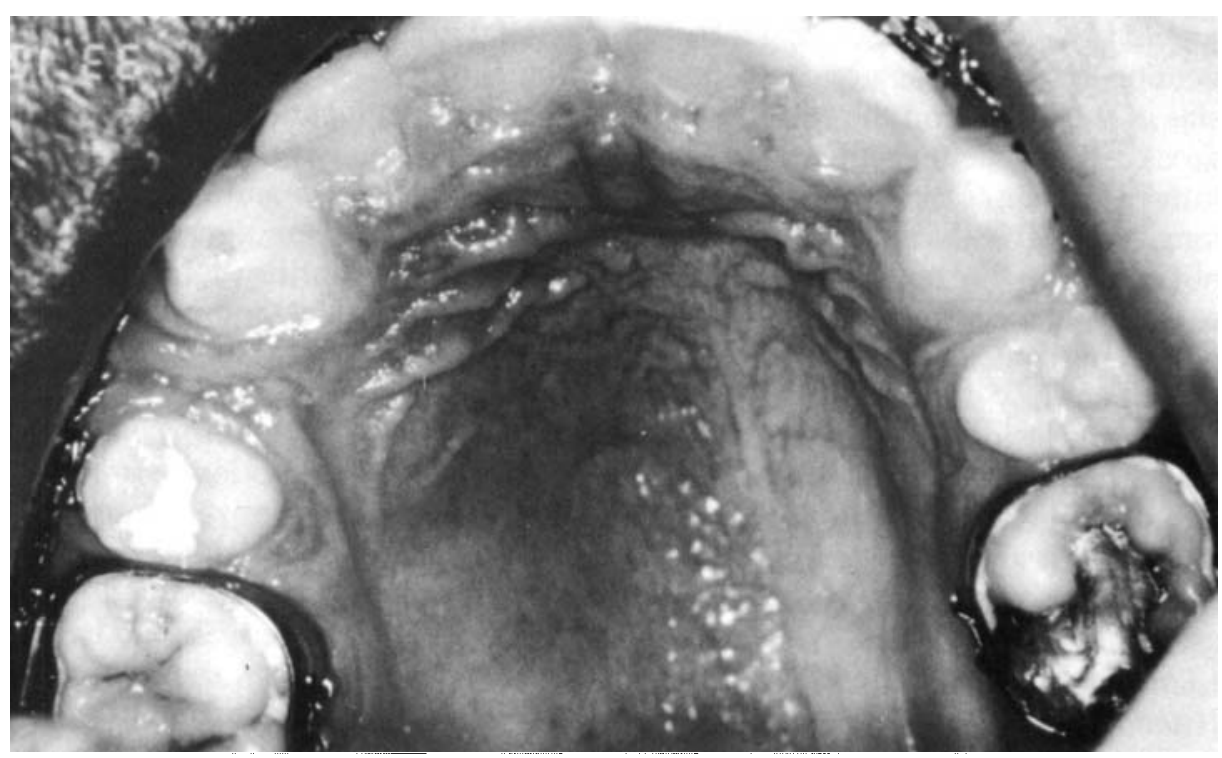

Figure 15. Case 5: Intra-oral palatal view showing bony and mucosal Involvement of the left side of the palate. 
procedures. Unfortunately, reports on successfully treated cases tend to be anecdotal, and the wide variety of available treatment options highlights the limitations in treating these lesions. ${ }^{6-11}$

The natural history of hemangiomas is a very important factor influencing their management. In Cases 1 and 2, the natural history of the hemangiomas was rapid growth (proliferative phase) within the first 18 months of life, followed by a gradual regression (involution). Studies have reported complete resolution of hemangiomas in over $50 \%$ of children by the age of 5 years, with further improvement occurring in the remaining cases up to age 10 to 12 years. Surgical intervention is, therefore, delayed as long as possible. The rate of regression of these lesions, however, cannot be predicted. Regression or involution is not related to gender, age, duration of the proliferative phase, site, size, or appearance of the hemangioma. These findings may lead to dilemmas in the timing of surgical treatment, particularly in facial anomalies where esthetics is a major consideration. ${ }^{12}$

In the cases illustrated, surgical intervention was carried out to improve esthetics in Cases 1, 2, and 5 , and to stop bleeding in Case 3. Case 4 opted to avoid any treatment. It was likely that the hemangioma in Case 1 would involute; early surgical intervention proved necessary due to the size of the lesion and the resulting gross esthetic disfigurement. Surgical intervention in Case 2 was delayed as long as possible. Early surgery was not an option. Fortunately, involution of the lesion occurred, and the successful outcome of the case was almost entirely due to natural regression of the lesion. The surgery that was carried out was done primarily to reduce the large amount of redundant skin tissue which had arisen due to the expansive nature of the hemangioma.

Case 3 also benefited from natural regression of the lesion; only a very pale pink lesion remained on the right cheek. The intra-oral maxillary cavernous hemangioma has been treated by a combination of surgical reduction, ligation procedures, and cryosurgery. The medical histories of Cases 4 and 5 resembled developmental vascular malformations, and these two cases showed no improvement with age. Case 4 opted to avoid surgery, and Case 5 has just begun a prolonged course of laser therapy whose successful outcome must be considered guarded.

In the two patients with unilateral cavernous hemangiomas of the maxilla, where orthodontic treatment was carried out, no serious complications have arisen to date. Interestingly, tooth movement in these highly vascular regions has progressed, though surprisingly at a slower rate than in the normal maxillary contralateral sides. With the greater vascularity and less bone in these regions, the opposite was expected. There have been no previous scientific reports concerned with the movement of teeth through bony areas with hemangiomas. It is difficult, therefore, to determine whether this finding of slow tooth movement is accidental or a feature of these lesions.

\section{Conclusions}

These cases illustrate the diverse clinical presentations of facial developmental vascular anomalies. From a dental perspective, it is important to identify their location and extent. Bony involvement of hemangiomas of the maxilla or mandible places a patient at risk of emergency hemorrhage. Early identification of these oral lesions is vital, and preventive dental care should be instituted to avoid the need for emergency dental procedures that would place the patient at risk. Orthodontic treatment should also be minimized. In those instances where orthodontic treatment is essential, a nonextraction approach is preferred. Should extractions prove necessary, they should be carried out in a hospital environment where emergency hemorrhage can be dealt with.

The authors wish to thank Dr. Jane Davies, Consultant Orthodontist, South Eastern Health Board, for her help in collecting data for these case reports, and also Colleen Gaffey for assisting with this article.

Dr. McNamara is Consultant Orthodontist, Western Health Board, Merlin Park Regional Hospital, Galway, Ireland. Dr. Trotman is Assistant Professor, Orthodontic Department, University of Michigan, Ann Arbor. Dr. Hewson is Principal Dental Officer,

Roscommon, Ireland. Dr. Walshe is Principal

Dental Officer, Mayo, Ireland. Correspondence should be addressed to Dr. McNamara.

1. Muir's textbook of pathology. Anderson JR, editor. 12th ed. London: Edward Arnold, pp 1439-41, 1985.

2. Gorlin RJ, Cohen MM, Levin LS. Syndromes of the head and neck. 3rd ed. Oxford, England: Oxford University Press, pp 323-32, 1990.

3. Jones KL. Smith's recognizable patterns of human malformation. 4th ed. Philadelphia, PA: W.B. Saunders, pp 442-3, 1988.

4. Mullikan JB, Young AE. Vascular birthmarks: hemangiomas and malformations. Philadelphia, PA: W.B. Saunders, pp 4162, 1988.

5. Wisnicki JL. Hemangiomas and vascular malformations. Ann Plast Surg 12:41-59, 1984.

6. Tal H. Cryosurgical treatment of hemangiomas of the lip. Oral Surg Oral Med Oral Pathol 73:650-4, 1992.

7. Barak S, Katz J, Kaplan I. The $\mathrm{CO}_{2}$ laser in surgery of vascular tumors of the oral cavity in children. J Dent Child 58:293-6, 1991.

8. Nixon H, O'Donnell B. The essentials of pediatric surgery. 3rd ed. London: Heinemann, pp 93-7, 1976.

9. Baurmash $\mathrm{H}$, DeChiara S. A conservative approach to the management of orofacial vascular lesions in infants and children: Report of cases. J Oral Maxillofac Surg 49:1222-5, 1991.

10. Brawner G, Schliftman A, Cosman B. Evaluation of argon laser surgery in children under 13 years of age. Plast Reconstr Surg 87:37-43, 1991.

11. Greene LA, Freedman PD, Friedman JM, Wolf M. Capillary hemangioma of the maxilla. A report of two cases in which angiography and embolization were used. Oral Surg Oral Med Oral Pathol 70:268-73, 1990.

12. Ezekowitz RA, Mulliken JB, Folkman J. Interferon alfa-2a therapy for life threatening hemangiomas of infancy. J Med 326:1456-63, 1992. 